\title{
Concepciones de los docentes sobre el tratamiento de las competencias a través de los videojuegos en el aula de ciencias
}

\author{
Antonio Alejandro Lorca Marín (D), José María Cuenca López $\mathbb{B}$, Bartolomé Vázquez \\ Bernal 1 , Emilio José Delgado Algarra \\ Rebut: 11/09/2019 Acceptat: 09/10/2019
}

\begin{abstract}
Resumen
La entrada de las TIC en general en el mundo académico es ya una realidad, el número de investigaciones, actividades divulgadas por los distintos medios tanto formales como no formales así lo demuestran. Los videojuegos (VJ) en particular juegan un papel importante en muchas de ellas. No obstante, el tratamiento de estos en el aula de ciencias experimentales (CCEE) es más escaso, y los que hay solo pertenecen a aquellas de corte divulgativo. Por tanto, existe una necesidad de profundizar sobre este recurso y en el área de CCEE en particular, con la finalidad de generar modelos de buenas prácticas y su implantación en el aula de ciencias.
\end{abstract}

En este estudio se presenta un estudio sobre las concepciones que tienen los docentes, tanto en formación inicial como en ejercicio, sobre el papel de los VJ en el tratamiento de las competencias y/o contenidos de ciencias. Se ha abordado bajo un corte cuantitativo dentro de un paradigma interpretativo, vislumbrándose la necesidad de acercarse a la subjetividad que existe en las competencias a través de los indicadores objetivos que nos permiten los contenidos.

Palabras claves: Videojuegos, Contenidos, Competencias, Didáctica, Formación del profesorado.

\begin{abstract}
The entrance of TIC in general in the academic world is a reality, the number of investigations, reported by the various formal and non-formal means activities prove it. Videogames in particular play an important role in many of them. However the treatment of these in the classroom experimental sciences (CCEE) is scarce, and there is only belong to those of divulging court. Therefore, there is a need to deepen this resource and CCEE area in particular in order to generate models of good practice and its implementation in the science classroom.

In this study, a study on the conceptions that teachers have both initial training and exercise on the role of VJ in the treatment of skills and / or science content is presented. It has been addressed under a quantitative court within an interpretive paradigm, glimpsing the need to approach the subjectivity that exists in competitions through objective indicators that allow us content.
\end{abstract}


The authors of the article conform a group specialized in educational innovation and have participated in different activities related to ICT. They have attended different courses and collaborated in 5 educational innovation projects. The work carried out by the group reckons that ICT can be applied to any course and teaching modality and that students positively value the usefulness of the interactive activities developed, mainly for their self-assessment process. Therefore, the prospects are to continue working in the development and implementation of new dynamic tools that support the teachinglearning process in the stages of assimilation of concepts, review and evaluation.

Key words: Videogames, Content, Skills, Teaching, Teacher.

\section{Introducción}

El proceso de enseñanza/aprendizaje (E/A) dentro del sistema educativo español, se caracteriza por una división clara entre el profesor que asume el papel único y exclusivo de experto. Desde esta distribución de roles, el docente siempre tiene el control pleno, seleccionando los contenidos, organizando y pautando el aprendizaje y el alumno asume una posición pasiva de receptor de estos. En contraposición, los estudiantes asumen un rol pasivo, limitándose a seguir las pautas mostrando muy poco control sobre su propio aprendizaje y participando de forma muy periférica en el proceso (Firth, Greaves \& Frydenberg, 2010). Investigaciones como las de Kornell, Castel y Eich, (2010) demuestran que existen formas adecuadas de aprender que contradicen las que hemos sostenido hasta este tiempo en los sistemas educativos.

El aprendizaje que se lleva a cabo en contextos formales, parece contraponerse al modelo de aprendizaje que despliegan nuestros niños en contextos informales, lúdicos, ubicuos (Sánchez \& Esnaola, 2014). Hasta el momento el rol del profesor estaba centrado en el proceso de enseñanza, actuando como un instructor de contenidos, donde los recursos están prediseñados y el uso de las TIC están al margen del currículo.

Se plantea que el profesor ponga énfasis en el aprendizaje, donde debe actuar como tutor, fomentando la autonomía del alumno, diseñando y gestionando sus propios recursos, donde la finalidad es hacer alumnos competentes y donde las TIC están integradas en el currículo. Para entender el uso que hacen los docentes de cualquier tecnología es importante saber no sólo qué conocimientos elabora o las barreras que lo limitan (Sánchez \& Galindo, 2018). Esto hace que el docente en ciencias domine no solo los contenidos, sino que tenga cualidades pedagógicas (habilidades didácticas, tutoría, técnicas de investigación-acción, conocimientos psicológicos y sociales...), habilidades instrumentales y conocimiento de nuevos lenguajes y características personales (madurez, seguridad, empatía...), de cara a afrontar la necesidad de conectar el aula de ciencias con la realidad en la que se mueve el alumno (Fernández, 2006). Se debe tratar de situar los aprendizajes en contextos lo más adecuados para que se genere esta reflexión, se genere esta conciencia y sean capaces de percibir la variedad de tipos de actividades y las posibilidades que tienen en el desarrollo de estos aprendizajes (De Pro, Sánchez \& Valcárcel, 2008).

La ciencia escolar plantea como objetivo general que los futuros ciudadanos conozcan los fenómenos del mundo, traten de entenderlos e interpretarlos empleando modelos teóricos, y desarrollen comportamientos adecuados y responsables en la sociedad (González, García \& Martínez, 2015). Por tanto, se exige del alumnado una actividad reflexiva necesaria para el desarrollo de la toma de conciencia y del pensamiento crítico (Martín \& Jiménez, 2015). El profesorado de ciencias debe establecer relaciones entre la ciencia y otras materias y aplicar estrategias metodológicas que ayuden a analizar problemas y elaborar sus propias respuestas ante éstos, a la par que enseña los conceptos, leyes y teorías 
científicas. Por lo que, dependiendo del modelo didáctico que cada profesor adopte (Perales, 2014), así será las actividades que plantee. El docente debe actuar de mediador para que el alumnado aprenda significativamente y desarrolle los contenidos conceptuales, procedimentales y actitudinales, que forman parte de la amplia variedad de competencias que va a necesitar en el mundo que va a encontrarse (De Pro, 2011; Sanmartí, 2010)

En este sentido, las TIC pueden ser un elemento valioso de las prácticas de enseñanza en general y de las ciencias en particular, añadiendo en términos de información el acceso, la flexibilidad, la diversidad de los medios de comunicación en su tratamiento y presentación (Martinho \& Pombo, 2009). Por lo que se hace necesario hacer hincapié en la relevancia que tienen los contenidos educativos digitales y su incorporación a las aulas.

Las relaciones entre los nuevos medios digitales y el aprendizaje, han sido objeto de estudio con una intensidad cada vez mayor en los últimos años. En este contexto, investigadores de la relevancia de Jenkins (2006), Gee (2008), Salen y Zimmerman (2004) o Prensky (2003), vienen reconociendo en los VJ, un enorme potencial educativo. Hasta tal punto que Prensky (2003) plantea que los VJ podrían ser el mecanismo con un mayor potencial de aprendizaje jamás conocido, ya que, si bien es posible ajustar el contenido de los VJ para que estén más en sintonía con los objetivos de aprendizaje o de socialización, el propio contenido del VJ en sí mismo, con sus reglas y su organización, más allá del contenido, posibilita una gran cantidad de aprendizajes positivos (Del Castillo et al., 2012).

Partimos de la idea de que los VJ contribuyen a sumergir a los menores en nuevas experiencias a partir de la simulación y la ficción, nuevos contextos para la generalización de los aprendizajes, protagonizando experiencias formales en contextos lúdicos asumiendo decisiones en la resolución de problemas sin riesgos reales. Como señala Lacasa (2011), se trata de aprender a experimentar el mundo de una forma nueva, viendo, sintiendo y operando sobre él; al actuar en un determinado dominio, se participa de las prácticas de un grupo social y se obtienen recursos para futuros aprendizajes y resolución de problemas en ese dominio.

Existe ciencia en las noticias (Jiménez-Liso et al., 2010), en las películas, en la televisión, en los centros y museos de ciencia, en Internet (De Pro, 2011) y como no también en los VJ. En este sentido, para González, García y Martínez (2015), es recomendable el uso de modelos explicativos, aunque éstos todavía carezcan del rigor científico que se irá adquiriendo con el tiempo.

Martinho y Pombo (2009) establecen que los VJ posibilitan la educación científica, colocan en la resolución de problemas contextualizados, actividades experimentales, trabajo colaborativo y un enfoque interdisciplinario de los problemas contemporáneos. Existe un gran potencial en los VJ a la hora de introducirlos en los procesos de aprendizaje para adquirir competencias y habilidades que favorezcan la alfabetización (Jenkins et al., 2006), ya que pueden, en un entorno lúdico y atractivo, aprender en un entorno estimulante (Bergna, 2013), realizar experimentos, a veces peligrosos en la vida real, cometer errores y aprender practicando. Con los VJ se puede repetir indefinidamente cualquier acción: si se realiza incorrectamente siempre se puede volver a intentar. Esa seguridad implica que el miedo al error se minimiza, hasta el punto que fallar puede ser una opción de juego (Marcos \& Santorum, 2012).

En lo que se refiere a los contenidos procedimentales, los relacionados con el «saber hacer» teórico y práctico, los alumnos y alumnas han de iniciarse en conocer y utilizar algunas de las estrategias y técnicas habituales en la actividad científica. La observación, la identificación y análisis de problemas, la recogida, organización y tratamiento de datos, la emisión de hipótesis, el diseño y desarrollo de la experimentación, la búsqueda de soluciones, y la utilización de fuentes de información, incluyendo en lo posible las proporcionadas por medios tecnológicos y la comunicación de los resultados obtenidos, son claves en la formación en ciencias. En este sentido, desde el ámbito educativo los VJ pueden convertirse en herramientas válidas para el desarrollo de determinadas habilidades y competencias, 
estimulando la creatividad y el aprendizaje $(G e e, 2008)$ mediante la utilización de entornos próximos a los estudiantes que posibiliten una máxima interacción y experimentación.

Por último, para el desarrollo de actitudes y valores, los contenidos seleccionados han de promover la curiosidad, el interés y el respeto hacia sí mismo y hacia los demás, hacia la naturaleza, hacia el trabajo propio de las CCEE y su carácter social, y la adopción de una actitud de colaboración en el trabajo en grupo. El acceso y tratamiento de la información sin barreras espacio-temporales y sin condicionamientos que trae aparejado el uso de los VJ, produce el surgimiento de un nuevo concepto de mediación educativa que afecta al modelo de relación entre el individuo, la cultura y la enseñanza.

Debido al carácter del área, los contenidos conceptuales, procedimentales y actitudinales formulados, y teniendo en cuenta los diferentes estilos de aprendizaje, la actividad del aula girará en torno a la realización de actividades en las que el alumnado debe tener participación. Appelbaum (2013) demuestra que los participantes que usan VJ de manera habitual captan más rápido los estímulos visuales, necesitan menos información para llegar a las mismas conclusiones, manifiestan mayor agilidad mental y son capaces de tomar decisiones con más rapidez que los que no juegan. Por ello, los juegos podrían caracterizarse como actividades que facilitan vivencias, y que se centran en experiencias en las que el jugador vive y se reconoce como un protagonista que interactúa con el medio, además de mejorar su pensamiento lógico y crítico y sus habilidades para resolver problemas (Higgins, 2001).

Si bien el VJ nace como elemento de diversión, ha sabido evolucionar adecuadamente y adaptarse a las necesidades del mundo para llegar a campos del conocimiento en los que, en un principio, era totalmente impensable. Los VJ deben generar curiosidad, creatividad, fomentar la resolución de problemas, reflexionar a través de un nuevo tipo de discurso interactivo y colaborativo, que alfabetiza en los nuevos medios (Lacasa, 2011).

Así, el modelo didáctico que se nos presenta abre un nuevo panorama ante el que los docentes y los sistemas educativos deben replantearse los nuevos espacios formativos, reflexionar sobre los contenidos educativos a trabajar, encontrar aquellas metodologías que se aproximan y garantizan una formación de calidad para ciudadanos del siglo XXI. El profesorado debe conocer qué ofrece el mundo de los VJ y su potencial como recurso didáctico. Dicha selección se puede ver influida por las actitudes y concepciones de los docentes a la hora de llevar a cabo iniciativas educativas con los VJ.

\section{Objetivo de la investigación}

El objetivo de nuestra investigación es el de conocer las concepciones que tienen los docentes en ejercicio y en formación inicial, sobre el uso didáctico de los VJ, así como la de conocer cuáles son las concepciones sobre los contenidos de ciencias a trabajar a través de los VJ. Así, las hipótesis de las que partimos son que existen diferencias entre los docentes en formación inicial y en ejercicio en cuanto a los contenidos a trabajar a través de los VJ y que tales diferencias están relacionadas con haber tenido formación específica en didáctica durante los procesos de formación inicial tanto para los docentes en formación como en ejercicio. 
UTE. Revista de Ciències de l'Educació Monogràfic 2019. Pàg. 71-87 traves de los videojuegos en el aula de ciencias

\section{Participantes}

En la presente investigación participan un total de 720 informantesa. Del total, 659 pertenecen al conjunto de los denominados "docentes en formación inicial", alumnos de la Facultad de Ciencias de la Educación de la Universidad de Huelva, tanto de Grado (TIPO I.1 N=570), como de Posgrado para la formación del profesorado de enseñanza secundaria (TIPO I.2 N=89). Además, aquellos pertenecientes al título de Grado se subdividieron en aquellos alumnos matriculados en la titulación de Grado de Maestro de Educación Infantil $(\mathrm{N}=243)$ o aquellos alumnos que pertenecían a la titulación del Grado de Educación Primaria $(\mathrm{N}=327)$.

Por otro lado, participan un total de 61 informantes pertenecientes al conjunto de docentes en ejercicio, es decir, docentes que desarrollan su labor profesional en aulas ordinarias, de los cuales 7, pertenecían al nivel de Educación Infantil, 32 al de Educación Primaria (TIPO II.1) y 22 docentes de Enseñanza Secundaria (TIPO II.2), de distintas especialidades relacionadas con las CCEE (Biología, Geología, etc.).

\section{Instrumento}

Nos proponemos acercarnos a conocer las concepciones sobre los contenidos a trabajar a través de los VJ. Por lo que, nos resulta interesante describir, analizar y por tanto conocer aquellas variables que inciden en el qué trabajar con los VJ. Dedicamos algunas cuestiones a conocer qué contenidos, qué competencias y qué conjunto de habilidades y destrezas, se favorecen con el uso de VJ en el aula. Destacar que se han seleccionado aquellas habilidades y destrezas relacionadas con el ámbito de las CCEE.

El instrumento de recogida de información fue el Cuestionario sobre actitudes y concepciones en los videojuegos y su implicación didáctica en el aula de Ciencias (CVJ/AC): características psicométricas y publicado en su totalidad en Lorca-Marín, Cuenca-López, y Vázquez-Bernal (2019). De este, en la presente investigación se han seleccionado aquellas variables de interés para el objetivo propuesto (Contenidos, Competencias, habilidades y destrezas).

\section{Resultados}

En la Tabla 1, se muestran los porcentajes y $x 2$-cuadrado de las variables relacionadas con los contenidos (conceptuales, procedimentales y actitudinales) y competencias a trabajar a través de los VJ en función del tipo de informante. La información de ésta se completa aportando la $\chi 2$ y el $p$-valor para cada uno de los cruces correspondientes.

\footnotetext{
${ }^{a}$ La codificación utilizada en el trabajo ha sido (I y II para referirse si el informante está en formación o en ejercicio, 1 y 2 para referirse al nivel, si son maestros (primaria) o profesores (secundaria)

TIPO I.1= maestros en formación, TIPO I.2= profesores en formación, TIPO II, 1= maestros en ejercicio, TIPO II.2= Profesores en ejercicio)
} 
Tabla 1: Clasificación de los participantes en función a las variables de estudio.

\begin{tabular}{|c|c|c|c|}
\hline \multicolumn{2}{|c|}{ En Formación (Universidad de Huelva) $(\mathrm{N}=659)$} & \multicolumn{2}{|c|}{ En Ejercicio (EABE) ( $N=61)$} \\
\hline $\begin{array}{c}\text { Alta Formación en Didáctica } \\
\text { Específica }\end{array}$ & $\begin{array}{c}\text { Baja Formación en Didáctica } \\
\text { Específica }\end{array}$ & $\begin{array}{c}\text { Alta Formación en Didáctica } \\
\text { Específica }\end{array}$ & $\begin{array}{c}\text { Baja Formación en Didáctica } \\
\text { Específica }\end{array}$ \\
\hline Grado de Infantil $(N=243)$ & & Ciclo de Infantil $(\mathrm{N}=7)$ & \\
\hline Grado de Primaria $(\mathrm{N}=327)$ & Posgrado $(\mathrm{N}=89)$ & Ciclo de Primaria $(\mathrm{N}=32)$ & Secundaria $(N=22)$ \\
\hline
\end{tabular}

Nota. Se representan el conjunto de criterios propuestos para la clasificación de los informantes.

Podemos observar como aparecen diferencias entre los dos grupos en cada uno de los valores que adquiere la variable contenidos. Así, el porcentaje de profesorado en ejercicio declara detectar más contenidos que los docentes en formación y de entre ellos, los procedimentales en mayor porcentaje que los conceptuales y los actitudinales. De cara a un análisis por tipo de informante según los criterios, en la tabla 2 , se muestra los porcentajes y $\chi^{2}$-cuadrado de estas mismas variables.

Tabla 2: Porcentajes y Ji-cuadrado de las variables relacionadas con los contenidos, competencias a trabajar a través de los VJs en función del tipo de informante

\begin{tabular}{|c|c|c|c|c|c|}
\hline Variable & Valores & $\begin{array}{c}\text { En Formación } \\
(\mathrm{N}=659)\end{array}$ & $\begin{array}{c}\text { En Ejercicio } \\
(\mathrm{N}=61)\end{array}$ & $x^{2}$ & $p$-valor \\
\hline & Conceptuales & $223(33.84 \%)$ & $37(60.66 \%)$ & 7.04 & .008 \\
\hline \multirow[t]{5}{*}{ Contenidos } & Procedimentales & $319(48.41 \%)$ & $53(86.89 \%)$ & 8.75 & .003 \\
\hline & Actitudinales & $234(35.51 \%)$ & $45(73.77 \%)$ & 12.43 & .000 \\
\hline & Com_ling & 191 (28.98\%) & $36(59.02 \%)$ & 10.24 & .001 \\
\hline & Raz_mat & $260(39.45 \%)$ & $41(67.21 \%)$ & 6.26 & .012 \\
\hline & Con_medio & $170(25.80 \%)$ & $43(70.49 \%)$ & 22.78 & .000 \\
\hline \multirow{5}{*}{ Competencias } & Cult_arti & $154(23.37 \%)$ & $52(85.25 \%)$ & 42.05 & .000 \\
\hline & Social_Ciud & $127(19.27 \%)$ & $33(54.10 \%)$ & 20.26 & .000 \\
\hline & Dig_Infor & $357(54.17 \%)$ & $33(54.10 \%)$ & .00 & .995 \\
\hline & Apren_Apren & $208(31.56 \%)$ & $45(73.77 \%)$ & 16.73 & .000 \\
\hline & Autonōmía & $240(36.42 \%)$ & $46(75.41 \%)$ & 12.48 & .000 \\
\hline
\end{tabular}

Al igual que en el caso anterior, existen diferencias a tener en cuenta de estos grupos y por tanto se debe proceder a un análisis más pormenorizado de cara a poder establecer estas diferencias. Para ello en la tabla 3, se ha procedido a analizar las posibles diferencias entre los cruces por pares (TIPO I.1/TIPO II.1 y TIPO I.2/TIPO II.2). No aparecen diferencias significativas en ninguno de los casos por lo que para simplificar las tablas y los datos no se ponen de manifiesto en el texto. 
UTE. Revista de Ciències de l'Educació Monogràfic 2019. Pàg. 71-87 traves de los videojuegos en el aula de ciencias

Tabla 3: Porcentajes y Ji-cuadrado de las variables relacionadas con los contenidos, competencias a trabajar a través de los VJs en función del tipo de informante según criterios de clasificación

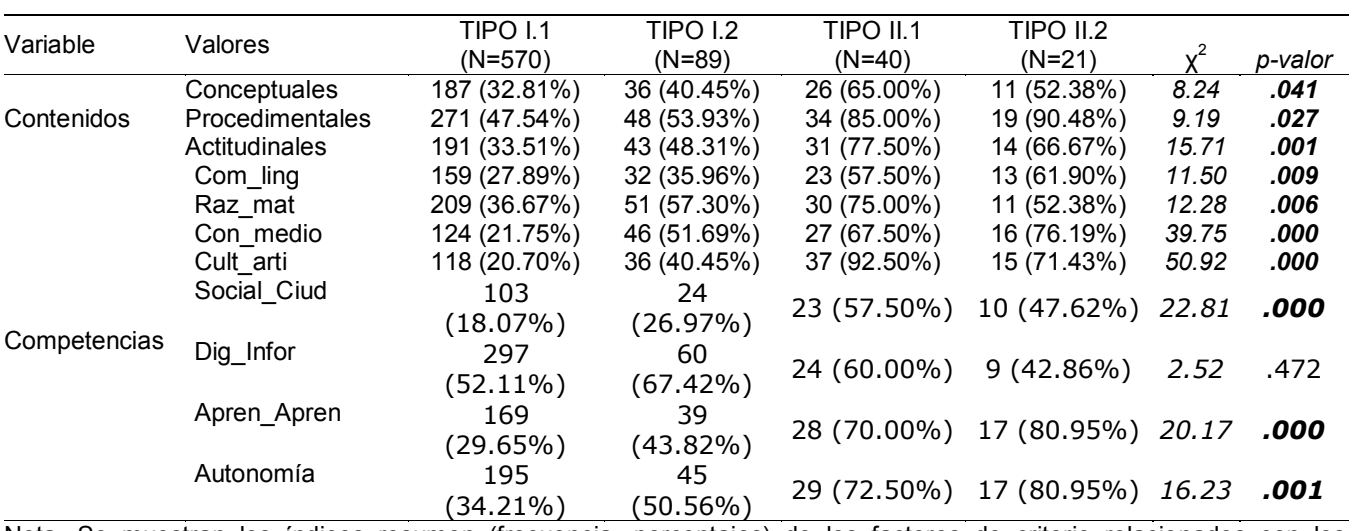

Nota. Se muestran los índices resumen (frecuencia, porcentajes) de los factores de criterio relacionados con los contenidos y competencias (en filas) distribuidos por tipo de informante según criterio (en columna). Se acompaña del grado de significación ( $p$-valor) de las diferencias de frecuencias a través de la prueba estadística Ji-cuadrado.

En cuanto a los contenidos, vemos que entre los maestros existen diferencias, siendo el porcentaje de los maestros en ejercicio mucho más alto en todos los casos que entre los que se encuentran en formación inicial. En cambio, estas diferencias dejan de aparecer cuando el análisis se realiza entre profesores (en formación/ en ejercicio), apareciendo en todos los casos, valores de porcentajes muy parecidos.

Cuando hablamos de competencias, vemos que existen diferencias sean cuales sean los grupos que se analicen, con excepción de la competencia digital y tratamiento de la información y con el cruce entre profesores (TIPO I.2/TIPO II.2), donde no aparece diferencia alguna. Por tanto, podemos deducir que las diferencias se encuentran entre los maestros en formación inicial y en ejercicio.

En la figura 1, se representan las distancias escalonadas de los valores z tipificados de las competencias que se pueden adquirir a través de los VJ. En ella podemos observar que, para los docentes en formación inicial, sea maestros (TIPO I.1) o profesores (TIPO I.2), asumen la competencia digital y tratamiento de la información como competencia a trabajar a través de estos. Sin embargo, cuando hablamos de docentes en ejercicio, varía sustancialmente, siendo la cultura artística la que adquiere gran protagonismo para los maestros en ejercicio (TIPO II.1) y aquellas con la autonomía personal y aprendizaje autónomo para los profesores (TIPO II.2). 


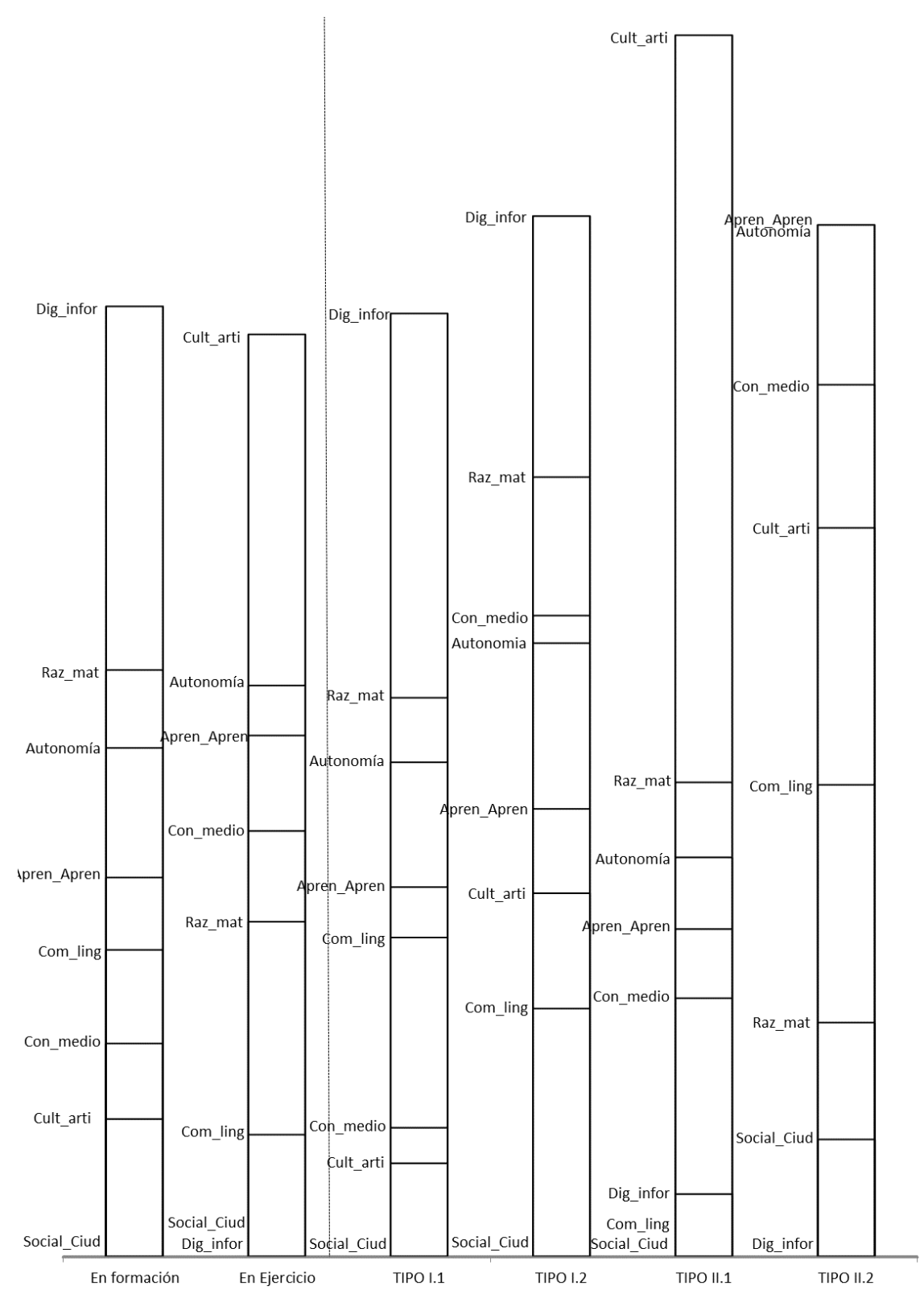

Figura 1. Distancia escalonada de las competencias adquiridas a través de los VJs.

Nota. Los valores representados se refieren a las diferencias de los índices tipificados z calculados a partir de las proporciones de elección en la muestra.

En cualquier caso, parece que la competencia que parece que los docentes no detectan a la hora de poner en juego los VJ como recurso es la de social y ciudadana.

Apréciese que la competencia Conocimiento y la interacción con el mundo físico y natural, adquiere porcentajes intermedios entre todos los informantes con excepción de los maestros en formación inicial cuyo valor desciende notablemente.

Como reflejo de los datos, debemos ir analizando de manera independiente cada una de las habilidades y destrezas propuestas, tanto por tipo de informante (ver tabla 4), como por tipo de informante según los criterios de clasificación (ver tabla 5). 
UTE. Revista de Ciències de l'Educació Monogràfic 2019. Pàg. 71-87 traves de los videojuegos en el aula de ciencias

Tabla 4: Ji-cuadrado de las variables relacionadas con los contenidos, competencias a trabajar a través de los VJs en función del tipo de informante según criterios de clasificación (cruce por pares)

\begin{tabular}{|c|c|c|c|c|c|}
\hline \multirow{2}{*}{ Variable } & \multirow{2}{*}{ Valores } & \multicolumn{2}{|c|}{ TIPO I.1 / TIPO II.1 } & \multicolumn{2}{|c|}{ TIPO I.2 / TIPO II.2 } \\
\hline & & $x^{2}$ & $p$-valor & $x^{2}$ & $p$-valor \\
\hline \multirow{3}{*}{ Contenidos } & Conceptuales & 6.83 & .009 & .38 & .539 \\
\hline & Procedimentales & 5.76 & .016 & 2.04 & .153 \\
\hline & Actitudinales & 11.44 & .000 & 0.68 & .410 \\
\hline \multirow{8}{*}{ Competencias } & Com_ling & 7.08 & .008 & 1.79 & .181 \\
\hline & Raz_mat & 8.16 & .004 & .05 & .827 \\
\hline & Con_medio & 19.33 & .000 & 1.06 & .303 \\
\hline & Cult_arti & 40.93 & .000 & 2.14 & .144 \\
\hline & Social_Ciud & 18.25 & .000 & 1.64 & .201 \\
\hline & Dig_Infor & .28 & .598 & 1.11 & .292 \\
\hline & Apren_Apren & 11.31 & .000 & 2.67 & .102 \\
\hline & Autonomía & 8.81 & .003 & 1.6 & .206 \\
\hline
\end{tabular}

Para los docentes en formación inicial, las habilidades y destrezas menos potenciadas por los VJ son: la alfabetización científica, la interacción, el pensamiento crítico y racional, la capacidad de experimentación, el fomento de la curiosidad y/o la participación, con un porcentaje de elección de menos del cincuenta por ciento. Por el contrario, parece ser que: la creatividad, la capacidad para la resolución de problemas, la atención y la agilidad mental, son las destrezas más valoradas por estos informantes. En cambio, para los docentes en ejercicio, prácticamente todas se muestran potenciadas cuando se pone en juego el uso de los VJ en el aula, con unos porcentajes de elección por encima del sesenta por ciento. Ambos grupos parecen coincidir en la opinión (puesto que no aparecen diferencias significativas en ambos grupos) que: la memoria, el análisis y la reflexión, son de las habilidades menos potenciadas por los VJ. Sin embargo, bajo la opinión de ambos grupos, las que más se potencian son: la resolución de problemas, la toma de decisiones, la creatividad y la agilidad metal. Para el resto de habilidades, parece haber una mayor valoración por parte de los docentes en ejercicio frente a los de formación inicial. Si esta misma descripción la presentamos para los tipos de informantes por criterios de clasificación (ver tabla 5), los resultados son similares.

Tabla 5: Porcentajes y Ji-cuadrado de las habilidades y destrezas relacionadas con el ámbito de las ciencias, que se pueden adquirir a través de los VJs en función del tipo de informante

\begin{tabular}{|c|c|c|c|c|}
\hline Variable & $\begin{array}{l}\text { En Formación } \\
(\mathrm{N}=659)\end{array}$ & $\begin{array}{c}\text { En Ejercicio } \\
(\mathrm{N}=61)\end{array}$ & $x^{2}$ & $p$-valor \\
\hline Creatividad & $397(60.24 \%)$ & $48(78.69 \%)$ & 1.74 & 0.188 \\
\hline Interacción & $165(25.04 \%)$ & $36(59.02 \%)$ & 14.85 & .000 \\
\hline Resolución de problemas & $422(64.04 \%)$ & $47(77.05 \%)$ & .83 & .364 \\
\hline Análisis y reflexión & $323(49.01 \%)$ & $40(65.57 \%)$ & 1.85 & .174 \\
\hline Curiosidad & $248(37.63 \%)$ & $42(68.85 \%)$ & 8.16 & .004 \\
\hline Búsqueda, estructuración y organización de la información & $301(45.68 \%)$ & $43(70.49 \%)$ & 4.28 & .039 \\
\hline Memoria & $284(43.10 \%)$ & $38(62.30 \%)$ & 2.87 & .090 \\
\hline Alfabetización científica & $130(19.73 \%)$ & $32(52.46 \%)$ & 17.84 & .000 \\
\hline Toma de decisiones & $384(58.27 \%)$ & $48(78.69 \%)$ & 2.19 & .138 \\
\hline Experimentación & $227(34.45 \%)$ & $41(67.21 \%)$ & 9.83 & .002 \\
\hline Atención & $426(64.64 \%)$ & $45(73.77 \%)$ & .41 & .521 \\
\hline Agilidad mental & $517(78.45 \%)$ & $54(88.52 \%)$ & .38 & .537 \\
\hline Participación & $272(41.27 \%)$ & $46(75.41 \%)$ & 8.57 & .003 \\
\hline Pensamiento racional & $218(33.08 \%)$ & $46(75.41 \%)$ & 15.97 & .000 \\
\hline Pensamiento crítico & $178(27.01 \%)$ & $40(65.57 \%)$ & 16.99 & .000 \\
\hline Aprender por sí mismo & $311(47.19 \%)$ & $51(83.61 \%)$ & 8.19 & .004 \\
\hline Trabajo individual & $321(48.71 \%)$ & $50(81.97 \%)$ & 6.71 & .009 \\
\hline
\end{tabular}


El grupo formado por maestros en formación inicial (TIPO I.1) son los que presentan porcentajes más bajos de acuerdos, en contraposición a los maestros en ejercicio (TIPO II.1) quienes presentan mayores porcentajes de elección.

Cuando se observan los datos en relación al cruce de docentes en formación inicial frente a docentes en ejercicio (TIPO I.1/TIPO II.2) y (TIPO I.2/TIPO II.2) (ver tabla 6), el análisis vuelve a no ser distinto a lo descrito anteriormente. Parece que tanto los docentes en ejercicio como en formación inicial, coinciden en ver la resolución de problemas, la toma de decisiones, la atención y la agilidad mental, como las habilidades más potenciadas. Por el contrario, la creatividad, el análisis, la reflexión y la memoria, son las habilidades menos promovidas por los VJ para ambos grupos. Para el resto de variables la valoración en la adquisición de habilidades a través de los VJ está más valorada por docentes en ejercicio que en formación inicial. Cuando comparamos las opiniones de los profesores (TIPO I.2/TIPO II.2) vemos como no existen diferencias significativas entre los porcentajes de elección de ambos grupos. Siendo muy elevada la valoración en el potencial que poseen los VJ para cada una de las habilidades analizadas.

Tabla 6: Porcentajes y Ji-cuadrado de las habilidades y destrezas relacionadas con las ciencias, que se adquieren a través de los VJs por tipo de informante según criterios de clasificación

\begin{tabular}{|c|c|c|c|c|c|c|}
\hline Variable & $\begin{array}{l}\text { TIPO I.1 } \\
(\mathrm{N}=570)\end{array}$ & $\begin{array}{l}\text { TIPO I.2 } \\
(\mathrm{N}=89)\end{array}$ & $\begin{array}{l}\text { TIPO II.1 } \\
(\mathrm{N}=40)\end{array}$ & $\begin{array}{l}\text { TIPO II.2 } \\
(\mathrm{N}=21)\end{array}$ & $x^{2}$ & p-valor \\
\hline Creatividad & $343(59.24 \%)$ & $54(60.67 \%)$ & $29(72.50 \%)$ & $19(90.48 \%)$ & 2.06 & .560 \\
\hline Interacción & $130(22.45 \%)$ & $35(39.33 \%)$ & $22(55.00 \%)$ & $14(66.67 \%)$ & 20.88 & .000 \\
\hline Resolución de Problemas & $356(61.49 \%)$ & $66(74.16 \%)$ & $31(77.50 \%)$ & $16(76.19 \%)$ & 1.78 & .620 \\
\hline Análisis y reflexión & $267(46.11 \%)$ & $56(62.92 \%)$ & $27(67.50 \%)$ & $13(61.90 \%)$ & 4.40 & .221 \\
\hline Curiosidad & $206(35.58 \%)$ & $42(47.19 \%)$ & $28(70.00 \%)$ & $14(66.67 \%)$ & 9.84 & .02 \\
\hline $\begin{array}{l}\text { Búsqueda, estructuración y organización de } \\
\text { la información }\end{array}$ & $252(43.52 \%)$ & $49(55.06 \%)$ & $30(75.00 \%)$ & $13(61.90 \%)$ & 5.78 & .123 \\
\hline Memoria & $246(42.49 \%)$ & $38(42.70 \%)$ & $27(67.50 \%)$ & $11(52.38 \%)$ & 3.23 & .358 \\
\hline Alfabetización científica & $102(17.62 \%)$ & $28(31.46 \%)$ & $22(55.00 \%)$ & $10(47.62 \%)$ & 23.08 & .000 \\
\hline Toma de decisiones & $317(54.75 \%)$ & $67(75.28 \%)$ & $30(75.00 \%)$ & $18(85.71 \%)$ & 5.25 & .154 \\
\hline Experimentación & $189(32.64 \%)$ & $38(42.70 \%)$ & $27(67.50 \%)$ & $14(66.67 \%)$ & 11.22 & .011 \\
\hline Atención & $366(63.21 \%)$ & $60(67.42 \%)$ & $30(75.00 \%)$ & $15(71.43 \%)$ & .50 & .919 \\
\hline Agilidad mental & $437(75.47 \%)$ & $80(89.89 \%)$ & $35(87.50 \%)$ & $19(90.48 \%)$ & 1.30 & .729 \\
\hline Participación & $229(39.55 \%)$ & $43(48.31 \%)$ & $32(80.00 \%)$ & $14(66.67 \%)$ & 9.61 & .022 \\
\hline Pensamiento racional & $179(30.92 \%)$ & $39(43.82 \%)$ & $32(80.00 \%)$ & $14(66.67 \%)$ & 18.61 & .000 \\
\hline Pensamiento crítico & $146(25.22 \%)$ & $32(35.96 \%)$ & $28(70.00 \%)$ & $12(57.14 \%)$ & 19.40 & .000 \\
\hline Aprender por sí mismo & $258(44.56 \%)$ & $53(59.55 \%)$ & $33(82.50 \%)$ & $18(85.71 \%)$ & 10.27 & .016 \\
\hline Trabajo individual & $267(46.11 \%)$ & $54(60.67 \%)$ & $32(80.00 \%)$ & $18(85.71 \%)$ & 8.61 & .035 \\
\hline
\end{tabular}

Nota. Se muestran los índices resumen (frecuencia, porcentajes) de las habilidades y destrezas relacionadas con el ámbito de las ciencias, que se pueden adquirir a través de los VJs (en filas) distribuidos por tipo de informante según los criterios de clasificación (en columna). Se acompaña del grado de significación ( $p$-valor) de las diferencias de frecuencias a través de la prueba estadística Ji-cuadrado.

último y en un análisis general, como se puede observar en la figura 2, las habilidades menos valoradas son la alfabetización científica y la interacción. Siendo la agilidad mental aquella que se considera como la habilidad más potenciada cuando el VJ se pone al servicio del proceso de E/A. 


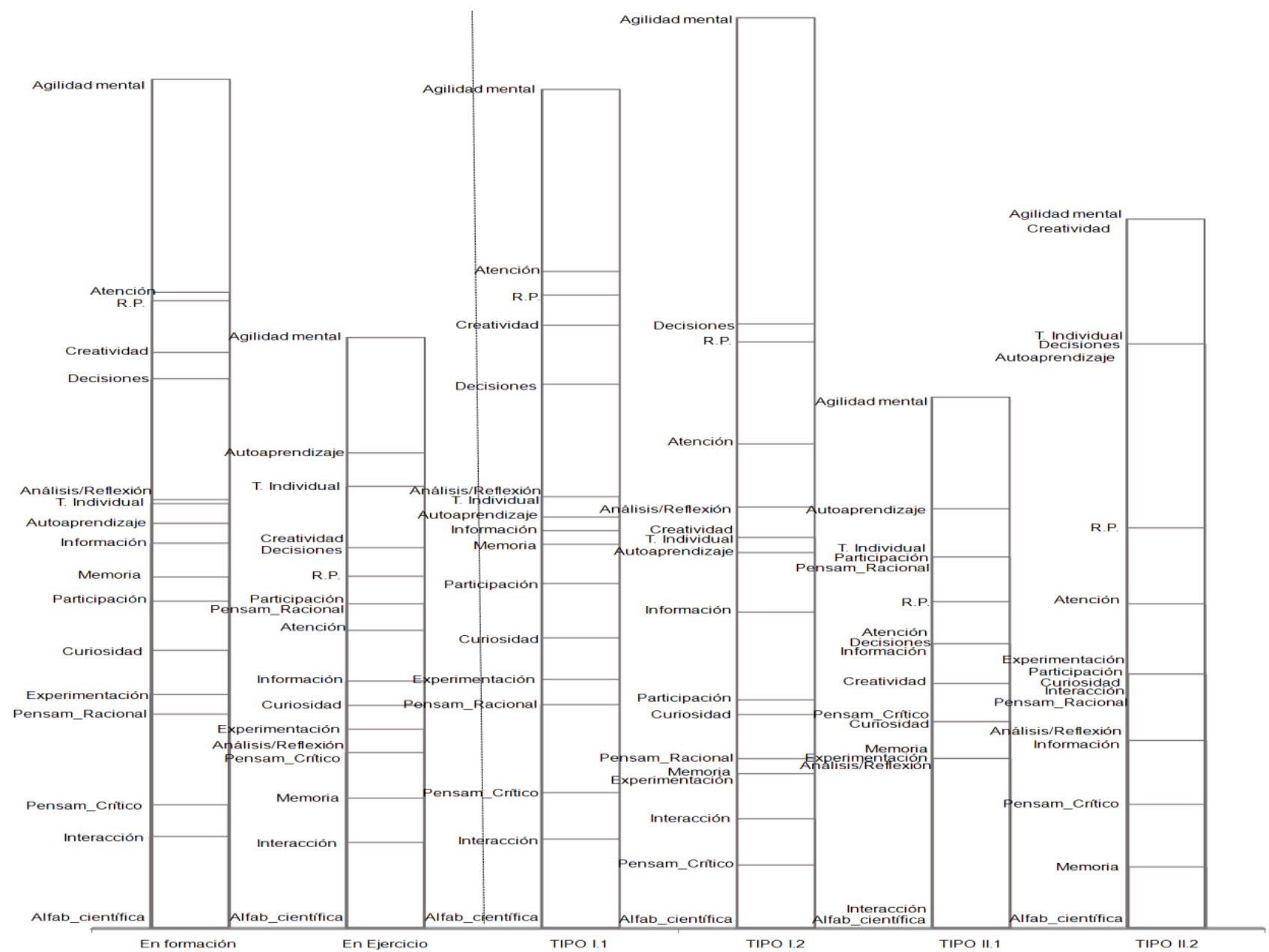

Figura 2. Distancia escalonada de las distintas habilidades y destrezas relacionadas con el ámbito de las ciencias, que se pueden adquirir a través de los videojuegos.

Nota. Los valores representados se refieren a las diferencia de los índices tipificados z calculados a partir de las proporciones de elección en la muestra.

\section{Discusión}

A la hora de poner de relieve qué tipo de contenidos se pueden trabajar desde el área de didáctica de las CCEE cuando usamos los VJ como recurso y que darán respuesta al segundo interrogante planteado en este objetivo, obtenemos que los docentes en ejercicio declaran detectar más contenidos que los docentes en formación inicial y de entre ellos, los procedimentales en mayor porcentaje que los conceptuales y los actitudinales.

En este sentido, parece que los docentes en ejercicio detectan, en contraposición a los docentes en formación inicial, todo tipo de contenidos de ciencias. Tienen presente la capacidad de los VJ para el aprendizaje de saberes, desarrollar habilidades y potenciar valores, por tanto, bajo su punto de vista conformaría su posible utilidad en el aula.

Se analizaron los resultados cruzando los docentes en formación inicial y los docentes en ejercicio, pero diferenciándolos según hubiesen tenido formación específica en didáctica o no. En este sentido se observa que los informantes más críticos con la adquisición de algún tipo de contenido a través de los 
VJ fueron los informantes en formación inicial y especialmente los que habían recibido formación específica en didáctica. Por el contrario, aquellos informantes que se encuentran en ejercicio y que, a su vez, en su periodo de formación cursaron asignaturas en didácticas específicas, son los que más apuestan por el uso de esta herramienta a la hora de adquirir contenidos. En el caso de este último grupo, valoraban especialmente la adquisición de los contenidos procedimentales ("Explorar los diferentes ecosistemas en una realidad virtual, con los que puedan interaccionar", "reflexionar sobre determinadas acciones en el medio ambiente", "clasificar los seres vivos", "a investigar, a buscar, a seleccionar elementos", "a experimentar, visualizando y practicando ciertos experimentos.", "a tomar decisiones".), seguidos de los actitudinales ("favorece la interacción entre la realidad social, cultural y natural...", "respeto por el medio ambiente y la naturaleza", "...fomenta la visión espacial de un planeta.") y por último, los conceptuales ("...conocer los ecosistemas y aquellos elementos que los conforman", "...los seres vivos, diferencias y similitudes." "...el cuerpo humano que permite trabajar de forma más visual y conocer nuestra anatomía por dentro...", "la mayor parte de contenidos relacionados con el sistema solar, la materia, energía, animales, etc.", "El ciclo del agua. La diversidad natural y social. Parajes naturales. El universo. Las células. Los tipos de rocas."). Curiosamente, tanto los de formación inicial como en ejercicio con o sin formación en didáctica, coincidían en que son los contenidos procedimentales los que más adquirirían a través de los VJ, seguidos de los actitudinales. Los datos apuntan a que el conjunto de docentes, en su totalidad, son escéptico a la hora de ver el valor de los VJ en la adquisición de los contenidos conceptuales.

En este punto, debemos decir que hemos observado, en la mayoría de los trabajos consultados, una escasa actitud crítica a la hora de evaluar los VJ como recurso didáctico. Solo los trabajos de Aranda, Sánchez-Navarro y Tabernero (2009), con una muestra de alumnos de secundaria, concluyen que los VJ no van más allá de su vertiente más lúdica y de entretenimiento. Por el contrario, existen multitud de trabajos que avalan el efecto positivo que sobre los contenidos en general facilitan los VJ (Abella \& García, 2010; Amar, 2010; González \& Blanco, 2008; Lacasa, 2011; Revuelta \& Guerra, 2012).

La mayoría de los trabajos consultados encuentran efectos positivos a nivel procedimental y actitudinal. En relación a los contenidos procedimentales, Laniado y Pietra (2005) evidencian la influencia de los VJ a la hora de potenciar habilidades cognitivas, emocionales, y como instrumento de estudio. Revuelta y Guerra (2012) resaltan su valor en el desarrollo de la autonomía y de destrezas útiles para la individualidad. Bernat, (2008) subrayan su interés para el desarrollo de habilidades comunicativas. En cuanto a los contenidos actitudinales, los trabajos consultados resaltan aquellos referidos a la adquisición de seguridad personal (Bernat, 2008; Gómez del Castillo, 2007), relaciones sociales (Gómez del Castillo, 2007) y la crítica reflexiva y el desarrollo de actitudes (Bernat, 2008; Gros 2008).

Sin embargo, no se han encontrado evidencias claras que avalen el valor de los VJ a la hora de la adquisición de contenidos conceptuales (mucho menos en relación a contenidos del área de las CCEE) más allá de experiencias puntuales docentes (Abella \& Gacía, 2010; González \& Blanco, 2008; Kahne et al., 2009; Lacasa, 2011). Por tanto, no podemos concluir con rigurosidad los beneficios de los VJ en este aspecto.

Otro de los elementos que hemos analizado por su interés educativo y como elemento curricular, son las Competencias. Cuando hablamos de competencias que los informantes declaran observar a la hora de trabajar con VJ en el aula, entre los docentes en formación inicial, con excepción de la competencia digital y tratamiento de la información, el resto en ningún caso supera el cincuenta por ciento. Así y, por el contrario, lo que ocurre con los docentes en ejercicio es que en su totalidad todas las competencias que declaran son trabajadas a través del uso de los VJ.

Centrándonos con los profesores en ejercicio, se observa como la competencia Cultural y Artística es la más seleccionada de entre ellas, seguidas de la Autonomía e iniciativa personal y Aprender a aprender. 
Sin embargo y sorprendentemente, aparece como la menos fomentada en los VJ la Digital y tratamiento de la información, Social y ciudadana y Comunicación lingüística.

Cuando los datos fueron analizados en base a la formación específica en didáctica que tuviesen los informantes, parece haber cierta contradicción entre los que ejercen y los que están en periodo de formación. En este sentido, los docentes en ejercicio afirman que a través de los VJ, la posibilidad es más probable que se adquieran la competencia Cultural y artística, Razonamiento matemático y Autonomía e iniciativa personal. Sin embargo, para los docentes en formación inicial es la competencia Digital y tratamiento de la información, Razonamiento matemático y Conocimiento y la interacción con el mundo físico y natural serían las adquiridas a través de esta herramienta con mayor probabilidad. Por el contario, parece ser que la competencia Social y ciudadana y Comunicación lingüística, son las que menos aprecian.

Como se ha podido comprobar, las opiniones sobre las competencias que se adquieren a través de los VJ, para los distintos tipos de docentes es muy heterogénea y no permite extraer un perfil claro de cómo los VJ pudieran favorecer la adquisición de competencias en la docencia. Pero estas diferencias e inconsistencias en las respuestas de los informantes, las entendemos como un problema derivado de la propia definición (Perales et al., 2014) y conceptualización de la idea de competencia. Es decir, cuando les preguntábamos a los informantes por las competencias, desde nuestro punto de vista, tenemos serias dudas sobre si los informantes tenían una idea univoca y unificada de la concepción de competencia sobre la que se consultaba. El término competencia en general y su clasificación en particular, genera una visión arbitraria y poco precisa de su propio significado, de ahí que se pudiesen explicar la variedad de respuestas obtenidas.

La búsqueda de evidencias independientes en la literatura, tampoco arroja luz en este punto. Todos los autores consultados (Díaz, 2013; Frey, 2012; Gros, 2008; Padilla-Zea et al., 2011, 2015;) coinciden en exaltar la importancia del aprendizaje por competencias como vértice en la gestión de los procesos educativos. Todos estos autores concurren que el proceso de E/A no debe centrarse en la evaluación de conocimientos, sino en la adquisición de competencias, es decir, la consecución de habilidades y destrezas que desarrolla y adquiere un alumno para comprender, transformar e interaccionar de manera efectiva con el mundo que lo rodea. Sin embargo, cuando se ponen en juego la evaluación de estas competencias nos encontramos evidencias específicas entorno a ellas. La literatura consultada engloba sus resultados y conclusiones en torno a habilidades, destrezas y actitudes específicas adquiridas a través de los VJ. No existe hasta el momento una evaluación en profundidad y sistemática de la adquisición de competencias de manera general y menos en el campo de las CCEE a través de este recurso.

Para Perales (2014), la enorme variedad de definiciones ha provocado un doble problema: el carácter polisémico del término y la existencia de diversos enfoques, de modo que resulta complicado hablar de definiciones consensuadas universalmente. Lo que sí cabe hacer es identificar aquellas características que le son propias y que nos permiten diferenciarlo de otros conceptos. Por tanto, no sería aventurado inferir que al igual que los autores se acercan a la competencia a través de las habilidades y destrezas, que los docentes que han participado en nuestro hayan realizado un análisis análogo. Es posible que éstos, asocien la adquisición de las competencias a una serie de habilidades. Es decir, la interpretación que hacen de cada competencia, se basa en la habilidad que asocien a ésta y por tanto varíe según el sujeto. A la vez, cuando se le solicita que lo contextualice en el campo de los VJ, cada sujeto lo interpreta según el VJ que conozca, de manera que es posible que la incoherencia se deba a la falta de concreción y precisión que lleva consigo el propio concepto de competencia, así como su contextualización.

La idea anterior se confirma cuando utilizamos términos más claros y unívocos como habilidades y destrezas por la que se consultaron. En este caso, se obtuvieron un acuerdo mayor y coherencia entre los distintos grupos. De esta forma, parece que los docentes en formación inicial y en ejercicio, independientemente de la formación específica en didáctica que tuviesen, coinciden que las habilidades 
más potenciadas por los VJ son: la agilidad mental, la atención, la resolución de problemas, el trabajo individual y la toma de decisiones. Por el contrario, la alfabetización científica, la interacción, la memoria, el pensamiento crítico y racional, el análisis y reflexión, la experimentación y curiosidad, son las destrezas menos potenciadas a ojos de los informantes.

En esta misma dirección, encontramos una amplia diversidad de trabajos que subrayan el uso de los VJ y su importancia en la adquisición de habilidades y destrezas del tipo, concentración, motoras, verbales, matemáticas, visuales y de resolución de problemas (Padilla et al., 2015; Sánchez \& Esnaola, 2014).

\section{Conclusiones}

A la hora de identificar los contenidos y competencias que se trabajan a través de los VJ, se aprecian en los resultados una relación directa con la experiencia docente. Los docentes en ejercicio identifican y enumeran un mayor número de competencias, contenidos y habilidades que puedan ser adquiridas por esta herramienta.

Además, el hecho de haber recibido una formación específica en didáctica, potencia esta relación. De manera que los docentes que, en su proceso de formación inicial recibieron formación en didáctica específica y que actualmente se encuentran en ejercicio, presentan mayor predisposición a encontrar competencias y contenidos.

Sin embargo, este resultado debe ser matizado. Respecto a los contenidos, esta diferencia entre grupos desaparece cuando los contenidos son diferenciados por la formación en didáctica.

Existe unanimidad entre todos los grupos en el potencial de los VJ a la hora de trabajar contenidos procedimentales, seguidos de los actitudinales. Solo de manera marginal se valoran los VJ como una herramienta práctica para trabajar contenidos conceptuales. Se observa que los informantes más críticos con la adquisición de algún tipo de contenido, a través de los VJ, fueron los informantes en formación inicial y que habían recibido formación específica en didáctica. Por el contrario, aquellos informantes que se encuentran en ejercicio y que, a su vez, en su periodo de formación cursaron asignaturas en didácticas específicas, son los que más apuestan por el uso de esta herramienta a la hora de adquirir contenidos.

Respecto a las competencias, como se evidenció, las opiniones son muy heterogéneas de como los VJ pueden favorecer la adquisición de las mismas. A pesar de coincidir nuestros resultados con los aportados desde la literatura científica sobre el valor de las competencias como eje central de los procesos educativos, hemos identificado un problema derivado de la propia definición y conceptualización de la idea de competencia. Es decir, desde nuestro punto de vista, tenemos serias dudas sobre si existe una idea unívoca y unificada de la concepción de competencia. El término competencia en general y su clasificación en particular, genera una visión arbitraria y poco precisa de su propio significado. Además, no sería aventurado inferir que, al igual que muchos autores se acercan a la competencia a través de las habilidades y destrezas, los docentes que han participado en nuestro estudio hayan realizado un análisis análogo. Es posible que éstos, asocien la adquisición de las competencias a una serie de habilidades. Por lo tanto, la interpretación que hacen de cada competencia, se basa en la habilidad que asocien a ésta y por tanto varíe según el sujeto.

La idea anterior se confirma cuando utilizamos términos más claros y unívocos como habilidades y destrezas por la que se consultaron. En este caso, se obtuvieron un acuerdo mayor y coherencia entre los distintos grupos. De esta forma, parece que los docentes en formación inicial y en ejercicio, independientemente de la formación específica en didáctica que tuviesen, coinciden que las habilidades 
más potenciadas por los VJ son: la agilidad mental, la atención, la resolución de problemas, el trabajo individual y la toma de decisiones. Por el contrario, la alfabetización científica, la interacción, la memoria, el pensamiento crítico y racional, el análisis y reflexión, la experimentación y curiosidad, son las destrezas menos potenciadas a ojos de los informantes.

Por tanto, podemos concluir que a las competencias debemos acercarnos a través de los contenidos que se subordinan en estas y se hace necesaria su incardinación a través de indicadores que las definen.

\section{Referencias bibliográficas}

Abella, L. E. \& García, A. (2010). El uso de videojuegos para la enseñanza de las ciencias, nuevos desafíos al papel docente. Revista EDUCyT, (2), 19-32.

Amar, V. (2010). La educación en medios digitales de comunicación. Pixel-Bit. Revista de Medios y Educación, (36), 115-124.

Appelbaum, L. G., Cain, M. S., Darling, E. F. \& Mitroff, S. R. (2013). Action video game playing is associated with improved visual sensitivity, but not alterations in visual sensory memory. Attention, Perception, $y$ Psychophysics, 75(6), 1161-1167.

Aranda, D., Sánchez-Navarro, J. \& Tabernero, C. (2009). Jóvenes y ocio digital. Informe sobre el uso de herramientas digitales por parte de adolescentes en España. Barcelona: Editorial UOC.

Area-Moreira, M. (2011). Las tecnologías de la información y la comunicación como recurso didáctico. En I. Cantón Mayo, y M. Pino-Juste (Coords.), Diseño y desarrollo del curriculum. Madrid: Alianza.

Bergna, P. (Productor). (2013) ¿Cómo aprender a aprender con los videojuegos? [Vídeo]. Disponible en http://www.youtube.com/watch?v=EuMLQcYIK5Y

Bernat, A. (2008). La construcción de conocimientos y la adquisición de competencias mediante el uso de los videojuegos. En B. Gros (Coord.), Videojuegos y aprendizaje, 93-112. Barcelona: Grao.

De Pro, A., Sánchez, G. \& Valcárcel, M. V. (2008). Análisis de los libros de texto de física y química en el contexto de la reforma LOGSE. Enseñanza De Las Ciencias, 26(2), 193-210.

De Pro, A. (2011). Conocimiento científico, ciencia escolar y enseñanza de las ciencias en la educación en secundaria. En A. Caamaño (Coord.), Didáctica de la física y la química (pp. 13-33). Barcelona: GraóMe.

Del Castillo, H., Herrero, D., García-Varela, A. B., Checa, M. \& Monjelat, N. (2012). Desarrollo de competencias a través de los videojuegos deportivos: Alfabetización digital e identidad. RED. Revista De Educación a Distancia, (33).

Díaz, A. (2013). Secuencias de aprendizaje. ¿Un problema del enfoque de competencias o un reencuentro con perspectivas didácticas? Profesorado, Revista de currículum y formación del profesorado, 17(3), 11 33. Disponible en http://www.ugr.es/ recfpro/rev173ART1.pdf

Fernández, A. (2006). Metodologías activas para la formación de competencias. Educatio siglo XXI, (24), 35-56. 
Firth, N., Greaves, D. \& Frydenberg, E. (2010). Coping styles and strategies: A comparison of adolescent students with and without learning disabilities. Journal of Learning Disabilities, 43(1), 77-85.

Frey, R. (2012). Computers games as preparation for future learning. En D. Ifenthaler, D. Eseryel, y X. Ge (Eds.), Assesment in game-based learning (pp. 431-451). London: Springer.

Gee, J. P. (2008). "Learning and games". The ecology of games: Connecting youth, games, and learning. En K. Salen (Ed.), The ecology of games (pp. 21-40). Cambridge: MIT Press. doi:10.1162/dmal.9780262693646.021

Gómez del Castillo, M. (2007). Videojuegos y transmisión de valores. Revista Iberoamericana De Educación, 43(6), 1-10.

González, C. \& Blanco, F. (2008). Integrating and educational 3D game in Moodle. Journal Simulation and Gaming, 39(3), 399-413. doi: 10.1177/1046878108319585

González, C., García-Barros, S. \& Martínez, C. (2015) Qué contenidos y qué habilidades cognitivolingüísticas emplea el profesorado de primaria y secundaria en la enseñanza de la astronomía. Enseñanza de las Ciencias, 33(2), 71-89.

Gros, B. (2008). Videojuegos y aprendizaje. Barcelona: Graó.

Higgins, S. (2001). ICT and teaching for understanding. Evaluation y Research in Education, 15(3), 164171.

Hodson, D. (2003). Time for action: science education for an alternative future. International Journal of Science Education, 25(6), 645-670.

Jenkins, H. (2006). Fans, bloggers and gamers: Exploring participatory culture. New York: New York University.

Jenkins, H., Purushotma, R., Clinton, K., Weigel, M. \& Robison, A. (2006). Confronting the challenges of participatory culture: Media education for the $21^{\circ}$ Century. Chicago, IL: The MacArthur Foundation.

Jiménez-Liso, M. R., Hernández-Villalobos, L. \& Lapetina, J. (2010). Dificultades y propuestas para utilizar las noticias científicas de la prensa en el aula de ciencias. Revista Eureka Sobre Enseñanza y Divulgación De Las Ciencias, 7(1), 107-126.

Kahne, J., Middaugh, E. \& Evans, C. (2009). The civic potential of video games. Massuchesetts: Massachusetts Institute of Technology Press.

Kornell, N., Castel, A. D., Eich, T. S. \& Bjork, R. A. (2010). Spacing as the friend of both memory and induction in young and older adults. Psychology and Aging, 25(2), 498.

Lacasa, P. (2011). Los videojuegos. Aprender en mundos reales y virtuales. Madrid: Ediciones Morata.

Laniado, N. \& Pietra, G. (2005). Videojuegos, internet y televisión: Cómo evitar sus efectos perjudiciales en nuestros hijos. Barcelona: Oniro.

Lorca-Marín, A. A., Cuenca-López, J. Ma. \& Vázquez-Bernal, B. (2019). Cuestionario sobre actitudes y concepciones en los videojuegos y su implicación didáctica en el aula de Ciencias (CVJ/AC): 
características psicométricas. International Journal of Educational Research and Innovation (IJERI), 11, $101-120$

Marcos, M. D. M. \& Santorum, M. (2012). La narración del videojuego como lugar para el aprendizaje inmersivo. Revista de Estudios de Juventud, (98), 77-89.

Martínez, C. \& González, W. (2014). Concepciones del profesorado universitario acerca de la ciencia y su aprendizaje y cómo la promoción de competencias científicas en la formación de futuros profesores de biología. Enseñanza de las Ciencias, 32(1), 51-81.

Padilla-Zea, N., Gutiérrez, F. L., López-Arcos, J. R., Abad-Arranz A. \& Paderewski, P. (2011). Modeling storytelling to be used in educational video games. Computers in Human Behavior, 31, 461-474.

Padilla-Zea, N., Medina, N., Gutiérrez-Vela, F. L., Paderewski, P., López-Arcos, J. R., Núñez Delgado, M. P. $\&$ Rienda-Polo, J. (2015). Evaluación continua para aprendizaje basado en competencias: Una propuesta para videojuegos educativos. Revista Iberoamericana de Informática Educativa, (21), 25-38.

Perales, F. J., Burgos, O. \& Gutiérrez, J. (2014). El programa Ecoescuelas: Una evaluación crítica de fortalezas y debilidades. Perfiles Educativos, 36(145), 98-119.

Prensky, M. (2003). Digital game-based learning. Computers in Entertainment, 1(1), 21-21.

Revuelta, I. F. \& Guerra, J. (2012). ¿Qué aprendo con videojuegos? Una perspectiva de metaaprendizaje del videojugador. Revista de la Educación a Distancia, (33). Disponible en http://revistas.um.es/red/article/view/233161

Salen, K. \& Zimmerman, E. (2004). Rules of play: Game design fundamentals. Massachusetts: MIT Press.

Sánchez García, A.B. \& Galindo Villardón, P. (2018). Uso e integración de las TIC en el aula y dificultades del profesorado en activo de cara a su integración. Profesorado. Revista de Currículum y Formación de Profesorado, 22(3), 341-358.

Sanmartí, N. (2010). Competencias: ¿más burocracia o un constructo útil? Trabajo presentado en el II Congreso Internacional de Didácticas. La actividad del docente: Intervención, innovación, investigación, Girona, España. 